\title{
Discharge against Medical Advice in Pediatric Intensive Care Unit and Subsequent Events
}

\author{
Dadang Hudaya Somasetia, Susanto Isman, Nathania Wonoputri
}

Department of Child Health, Faculty of Medicine, Universitas Padjadjaran-Dr. Hasan Sadikin General Hospital

\begin{abstract}
Objective: The occurence of discharge against medical advice (DAMA) has impacted globally. Decisions of DAMA in pediatric patients are not made by the patients, but pose life-threatening consequences, especially for children treated in Pediatrics Intensive Care Unit (PICU). The present study aimed to identify the occurence of DAMA and subsequent events to the DAMA patients after treated in PICU of Dr. Hasan Sadikin General Hospital Bandung, Indonesia.
\end{abstract}

Methods: A retrospective study from medical records of DAMA patients from PICU of Dr. Hasan Sadikin General Hospital, Bandung, Indonesia, between April 2011 and April 2012.

Results: A total of 195 children aged 1-5 years were admitted. Of this number, $12(6.2 \%)$ were DAMA. Financial problems were the most common reason for DAMA, followed by parents' perception about their child's terminal condition. The majority of parents were laborer. Last parents' education level was mostly elementary school. Most patients who DAMA from PICU died in less than 24 hours. $\begin{array}{ll}\text { Received: } & \text { Conclusions: A considerable number of DAMA was found in our PICU. High } \\ \text { April 10,2014 } & \text { mortality occured after patients asked for DAMA from PICU. }\end{array}$ mortality occured after patients asked for DAMA from PICU.

Revised:

August 18, 2014

Keywords: Discharge against medical advice (DAMA), pediatric, pediatrics intensive care unit (PICU)

Accepted:

February 4, 2015

IJIHS. 2015;3(1):7-10

\section{Introduction}

Discharge against medical advice (DAMA) is a condition that occurs when a patient, or the parents or caregivers in the case of pediatric patients, decides to leave the hospital without approval of the treating physician..$^{1-4}$ Discharge against medical advice from hospitals may have negative effects on the well-being of the patients. ${ }^{5}$ Contrary to adults, the decision for DAMA in pediatric patients is made by the parents. ${ }^{2,5,6}$ Clinicians are often confronted by parents/caregivers who insist on DAMA even when it is obvious that such decision is detrimental to the health of their children, especially to those with critically condition with life threatening complications. This is

\footnotetext{
Correspondence:

Dadang Hudaya Somasetia, Department of Child Health, Faculty of Medicine, Universitas Padjadjaran-Dr. Hasan Sadikin General Hospital

Jl. Pasteur No. 38, Bandung, Indonesia

e-mail:vemma.hees@gmail.com
}

not only disturbing but also frustrating for clinicians in their efforts to ensure that the child's health is restored. Discharge against medical advice will prevent the patients from receiving full benefits of services rendered by a health facility, leading to a higher risk of complications, prolonged morbidities, higher readmission prevalence threat, and even the patient's life. ${ }^{1-4,6-8}$

The problem of DAMA has been reported globally, including DAMA problems among pediatric patients. ${ }^{3,6,9,10}$ The DAMA prevalence varies depending on geographical areas and population studies. The percentage of children who are DAMA have been reported to be 1.2$31.7 \% \%^{3,5,8,9}$

The rate of DAMA correlates inversely with the socioeconomic status: a relatively lower rate $(0.8 \%)$ was observed at a hospital serving primarily middle and upperclass populations, ${ }^{11}$ whereas a higher rate $(2.2 \%)$ was observed at a hospital serving disadvantaged urban areas. ${ }^{12}$ In the United States, 2 characteristics strongly 
linked to low socioeconomic status: the lack of health insurance and Medicaid eligibility, which are associated with an approximately 2 -fold increase in the likelihood of DAMA.11,12

Our experience at a third referral hospital providing care for a low income population revealed that the rates of DAMA at pediatric intensive care unit (PICU) was high. This may lead to medical and legal problems. The ethical problems often appear for physicians caring for patients with DAMA, especially in PICU. The present study aimed to evaluate the occurence of DAMA and subsequent events to the patients after being treated in PICU of Dr. Hasan Sadikin General Hospital, Bandung, Indonesia.

Table 1 Characteristics of Discharged against Medical Advice Patients in Pediatric Intensive Care Unit Dr. Hasan Sadikin General Hospital during the Period of April 2011April 2012

\begin{tabular}{ll}
\hline \multicolumn{1}{c}{ Variables } & n \\
\hline Gender & 5 \\
Boy & 7 \\
Girl & $6.3(1-18)$ days \\
Length of stay & \\
Reasons for discharged & \\
against medical advice & 9 \\
Financial problem & 3 \\
Terminal illness & \\
Parent's occupation & 5 \\
Laborer & 2 \\
$\quad$ Private employee & 4 \\
Entrepreneur & 1 \\
Unemployed & \\
Patient's father education & 7 \\
Elementary school & 2 \\
Junior high school & 2 \\
Senior high school & \\
Graduate & \\
Patient's mother education & \\
Elementary school & \\
Junior high school & \\
Senior high school & \\
Graduate & \\
\hline & \\
\hline
\end{tabular}

\section{Methods}

This was a retrospective descriptive study at PICU of Dr. Hasan Sadikin General Hospital, Bandung, Indonesia. The study population was patients who were admitted to PICU between April 2011 and April 2012. The information collected consisted of age, sex, main diagnosis, residence, hospital stay duration, reasons of DAMA, and conditions after DAMA. Parents' education and occupation data were extracted from the medical records. Data obtained were analyzed according to sex, age, main diagnosis, and reasons for DAMA.

\section{Results}

During the study period, 195 children between one month and 14 years old were admitted in PICU of Dr. Hasan Sadikin General Hospital. Of this number, $12(6.2 \%)$ were DAMA. The DAMA patients' characteristics are shown (Table 1). The DAMA patients consisted of 5 boys and 7 girls with a male-to-female ratio of 1:1.4. The average length of stay was $6.3(1-18)$ days of DAMA patients. The most frequently found reason of DAMA was financial problem, followed by parents perceiving their child's condition as terminal.

Table 2 Conditions after Discharged Against Medical Advice

\begin{tabular}{ll}
\hline \multicolumn{1}{c}{$\begin{array}{c}\text { Condition after Discharged } \\
\text { against } \\
\text { Medical Advice }\end{array}$} & n \\
\hline Death occuring less than 24 hours & 8 \\
Alive & 4 \\
\hline
\end{tabular}

The parents characteristics are shown (Table 2). The majority of parents were laborer. Most parents were mostly elementary school graduates. The conditions of the patients after DAMA from PICU are also shown. Most of the patients died in less than 24 hours after DAMA.

\section{Discussion}

A previous research has shown that DAMA has become one of the biggest preventable problems, contributing about $2 \%$ of hospital discharge. ${ }^{10}$ Understanding the reasons why certain patients choose to be DAMA from 
hospital is important because it creates an opportunity to identify those patients at higher risk for DAMA and early intervention can be implemented to prevent higher morbidity, mortality, and health care costs, ${ }^{13}$ especially in PICU. The followings have been shown consistently as having correlation with DAMA over time: lower socioeconomic class, ${ }^{8,12,14}$ younger age,, $7-9$ no insurance, ${ }^{15,16}$ and substance abuse. ${ }^{4,15,16}$

The rate of DAMA in pediatric wards has been reported in recent years $\mathrm{S}^{5,8,9,14,17}$ as ranging from $1.2 \%{ }^{9}$ to $31.7 \%,{ }^{17}$ but we did not find any data on the prevalence of DAMA from PICU in other literatures. This study shows that DAMA in our PICU was $6.2 \%$, which is similar to the range of DAMA prevalence in previous studies among pediatric patients. Our present study showed that the majority of DAMA patients were girls. It is in agreement with the study by Onyiriuka in Nigeria which reported a higher rate of DAMA in girls. ${ }^{14}$ The mean time of hospitalization in our study was 6.3 (1-18) days and 3 children were discharged based on their parents' decision within 48 hours after admission and the reason for DAMA was financial problem. Most parents' were laborers with low income and the education level of the parents was elementary school. This might impact their decision of DAMA for their children. Similar finding has also been reported by Onyiriuka ${ }^{14}$ that concluded nearly half of the parents of children that DAMA lacked of formal education and mostly belonged to the lower social class. Our study emphasized this conclusion.

The majority of published studies on DAMA focus on adult patients, indicating that the patients were poor, lacking family support, financially and socially disadvantaged, and with no health insurance coverage. ${ }^{16}$ In our study, the most common reason cited by the parents for DAMA was financial problem (75\%). Our result is different from the study conducted by Rodpeyma ${ }^{5}$ which found that the most common reason was perceived improvement of the child's illness. Financial problem was the last reason for DAMA in their study. However, the result of our study is in agreement with Onyiriuka, ${ }^{8,14}$ Ibekwe et. al, ${ }_{1}^{18}$ and Okechukwu ${ }^{19}$ stating that the financial factors are more relevant in countries where health services are not covered by insurance. ${ }^{4}$ Financial problem here does not only include the lack of health insurance to cover medical costs, but also the lack of financial mean for daily living cost of family member who stay in the hospital, waiting on the patients. Our study reemphasizes the effect of financial cost in determining health-care seeking behavior stated by other study. ${ }^{8}$

In our study, 8 of 12 patients with DAMA were under mechanical ventilator when the parents decided to DAMA. Most of these patients died in less than 24 hours after the DAMA. Conflicts over the maintaining or withdrawing the life support may occur among the relevant parties, including the patients, families, health care professionals, hospitals, and other "third parties." Most conflicts can be avoided by first considering the goals of therapy and the potential interventions to achieve the goals. When conflicts arise, it is important to consider both the principles that underlie ethical decisions and the quality of communications among the relevant parties. Information given during the informed consent process in DAMA is one of the most important elements of care for patient's parent to make the decision. In this study, the parents already received information from the physician about the possible events that may occur after withdrawing life support from their children and the decision was made by the parents after considering all the consequences.

The limitations of our study come from the fact that our study was conducted in a single health institute with a small sample size. A cross sectional study involving more health institutes will be needed in order to comprehend the risk factors of DAMA in this population. Comprehending the risk factors will enable the physician to develop a better prevention plan for DAMA. This study helps us to identify and understand the reasons for discharging children against medical advice since it is often dilemmatic for physicians when facing parent's decision to discharged their child against medical advice, especially when the patient still needs supporting devices such as mechanical ventilator and intensive observation to support the life of the patient.

In conclusions, a considerable number of DAMA is found in our PICU. The mortality rate after these patients are discharged against medical advice, as the subsequent event, is high. 


\section{References}

1. Macrohon CB. Pediatrician's perpectives on discharge against medical advice (DAMA) among pediatric patients: a qualitative study. BMC Pediatr. 2012;12(75):1-9.

2. Paul SB, Remorin RM. Discharge against medical adivice (DAMA)-a study. West London Med J. 2010;2(3):17-27.

3. Al-Sadoon M, Al-Shamousi K. Discharge against medical advice among children in Oman. Sultan Qabos Univ Med J. 2013;13(4):534-8.

4. Debono R, Paul SB, Heaton PA. Children discharged against medical advice. Nurs Times. 2013;109(31):20-1.

5. Roodpeyma S, Hosyeni SAE. Discharge of children from hospital against medical advice. Word J Pediatr. 2010;6(4):353-6.

6. Saravi BM, Zadeh ER, Siamian H, Yahghoobian M. Discharge against medical advice in the pediatric wards in Boo-ali Sina Hospital, Sari, Iran 2010. Acta Inform Med. 2013;21(4):2536.

7. Bahadori M, Raadabadi M, Salimi M, Ravangard R. Discharge againstmedical advice: a case study in a public teaching hospital in Tehran, Iran in 2012. Global J Health Sci. 2013;5(6):179-85.

8. Onyiriuka AN. Pediatric discharge against medical advice: experience from a Nigerian secondary healthcare institution. Med J Islam Repub Iran. 2011;25(4):194-9.

9. Okoromah CN, Egri-Okwaji MT. Profile and control measures for pediatrics discharges against medical advice. Niger Postgrad Med J. 2004;11:21-5.

10. Ibrahim SA, Kwoh CK, Krishnan E. Factors asociated with patients who leave acute-care hospitals against medical advice. Am J Public
Health. 2007;97(12):2204-8.

11. Weingart SN, Davis RB, Phillips RS. Patients discharged against medical advice from a general medicine service. J Gen Intern Med. 1998;13:568-71.

12. Jeremiah J, O'Sullivan P, Stein MD. Who leaes against medical advice? J Gen Intern Med. 1995;10:403-5.

13. Onukwugha E, Saunders W, Mullins CD, Pradel FG, Zuckerman M, Weir MR. Reasons for discharge against medical advice: a qualitative study. Qual Saf Health Care. 2010;19(5):420-4.

14. Onyiriuka AN. Discharge of hospitalized under fives against medical advice in Benin city, Nigeria. Niger J Clin Pract. 2007;10:200-4.

15. Aliyu ZY. Discharge against medical advice: sociodemographic, clinical and financial perpectives. Int J Clin Pract. 2002;56(5):325-7.

16. Alfandre DA. I'm going home: discharges against medical advice. Mayo Clin Proc. 2009;84(3):255-60.

17. Meremikwu MM, Ehiri JE, Nkanga DG, Udoh EE, Ikapatt OF, Alaje EO. Socioeconomic constraints to effective management of Burkitt's lymphoma in South Eastern Nigeria. Trop Med Int Health. 2005;10:92-8.

18. Ibekwe RC, Muoneke VU, Nnebe-Agumadu UH, Amadife MA. Factors influencing discharge against medical advice among paediatric patients in Abakaliki, South eastern Nigeria. J Trop Paediatr. 2009;55(1):39-41.

19. Okechukwu AA. Discharge against medical adivice in children at the University of Abuja Teaching Hospital, Gwagwalada, Nigeria. J Med Med Sci. 2011;2(7):949-54. 